\title{
Optically Trapped Droplets of Liquid Crystals as Flexible, Tunable Optofluidic Microcavities
}

\author{
Alexandr Jonášs, ${ }^{\text {a }}$ Zdeněk Pilát, ${ }^{\mathrm{b}}$ Jan Ježek, ${ }^{\mathrm{b}}$ Silvie Bernatová, ${ }^{\mathrm{b}}$ Pavel Zemánek, \\ Mehdi Aas, ${ }^{\mathrm{c}}$ and Alper Kiraz ${ }^{\mathrm{c}}$ \\ ${ }^{a}$ Department of Physics, Istanbul Technical University, Maslak, 34469 Istanbul, Turkey \\ ${ }^{b}$ Institute of Scientific Instruments of the CAS, v.v.i., Královopolská 147, 61264 Brno, \\ Czech Republic \\ ${ }^{c}$ Department of Physics, Koç University, Rumelifeneri Yolu, Sariyer, 34450 Istanbul, \\ Turkey \\ *ajonas@itu.edu.tr
}

\begin{abstract}
We demonstrate reversible spectral tuning of optofluidic microcavities formed by optically trapped, dye-doped emulsion droplets of liquid crystals by changing ambient temperature or exposing the droplets to AC electric field of varying magnitude.
\end{abstract}

OCIS codes: (140.3945) Microcavities; (160.3710) Liquid crystals; (300.2530) Fluorescence, laser-induced; (350.4855) Optical tweezers or optical manipulation

\section{Introduction}

Optofluidics utilizes optical and mechanical properties of fluids for creating unique reconfigurable optical systems inconceivable with conventional solid-state materials [1,2]. Active fluid-based optical resonators (cavities) containing a suitable gain medium are among the most critical components of optofluidic systems as they represent a prerequisite for developing on-chip integrated sources of laser light [3-5]. Liquid microdroplets suspended in a lowrefractive index environment act as self-assembled optical cavities hosting high-quality optical resonances whispering gallery modes (WGMs) [6]. Droplets of liquid crystals (LC) - in contrast to ordinary liquids - show optical anisotropy and birefringence that can be controlled externally by electric field or changes of the ambient temperature. Thus, LC droplets are attractive for implementing tunable optofluidic cavities $[7,8]$.

We report on the development and characterization of tunable optofluidic resonators based on dye-doped LC emulsion droplets suspended in an immiscible host liquid with the refractive index smaller than the refractive index of the LC material, thus supporting WGM resonances. The contrast of refractive index necessary for excitation of WGMs in the droplets also enables stable spatial confinement of the studied droplets in optical tweezers which adds large flexibility to the spectroscopic experiments. We show that the WGM emission spectrum of an LC dropletbased resonator can be largely and (almost) reversibly tuned by controlled changes of the ambient temperature that induce phase transitions in the LC droplets. We also demonstrate the possibility of tuning reversibly the WGMs of LC droplet cavities by modifying the spatial profile of the effective refractive index of the cavity via external AC electric field of varied magnitude and frequency. Our results indicate feasibility of these approaches for creating miniature tunable sources of coherent light that can be integrated into microfluidic chips.

\section{Materials and methods}

Experimental setup used for the WGM spectroscopy of optically trapped emulsion droplets of liquid crystals was described previously [9]. Spectroscopic experiments were carried out in specially designed sample chambers that allowed adjusting the optical properties of the studied LC emulsion droplets by external stimuli (temperature changes, AC electric field) in parallel with confinement of the droplets in a single-beam optical trap. During the experiment, actual internal structure of the trapped LC droplet could be visualized by polarization microscopy.

Our working emulsions consisted of 5CB liquid crystal (refractive index in isotropic phase $n_{\mathrm{LC}}=1.58$ at $640 \mathrm{~nm}$ ) containing $1 \% \mathrm{w} / \mathrm{w}$ Nile Red fluorescent dye and emulsified in deionized water $\left(n_{\text {host }}=1.33\right)$ in the presence of 4 $\mathrm{mM}$ sodium dodecyl sulfate surfactant. At room temperature around $23^{\circ} \mathrm{C}$, this material combination leads to LC emulsion droplets with radial orientation of the molecular director [8]. The emulsion droplets were prepared by manual shaking of the liquid mixture and subsequently loaded into the sample chamber immediately before the experiments.

\section{Temperature-induced tuning of emission spectra of LC droplets}

Figs. 1(a),(b) present typical fluorescence emission spectra recorded from an optically trapped LC droplet of diameter approximately $13.5 \mu \mathrm{m}$ at different stages of the heating/cooling cycle, with the actual sample temperature 
indicated in the plot headings. Inset in each spectrum shows the corresponding image of the studied droplet acquired using polarization microscopy which reports on the internal organization of LC molecules within the droplet.
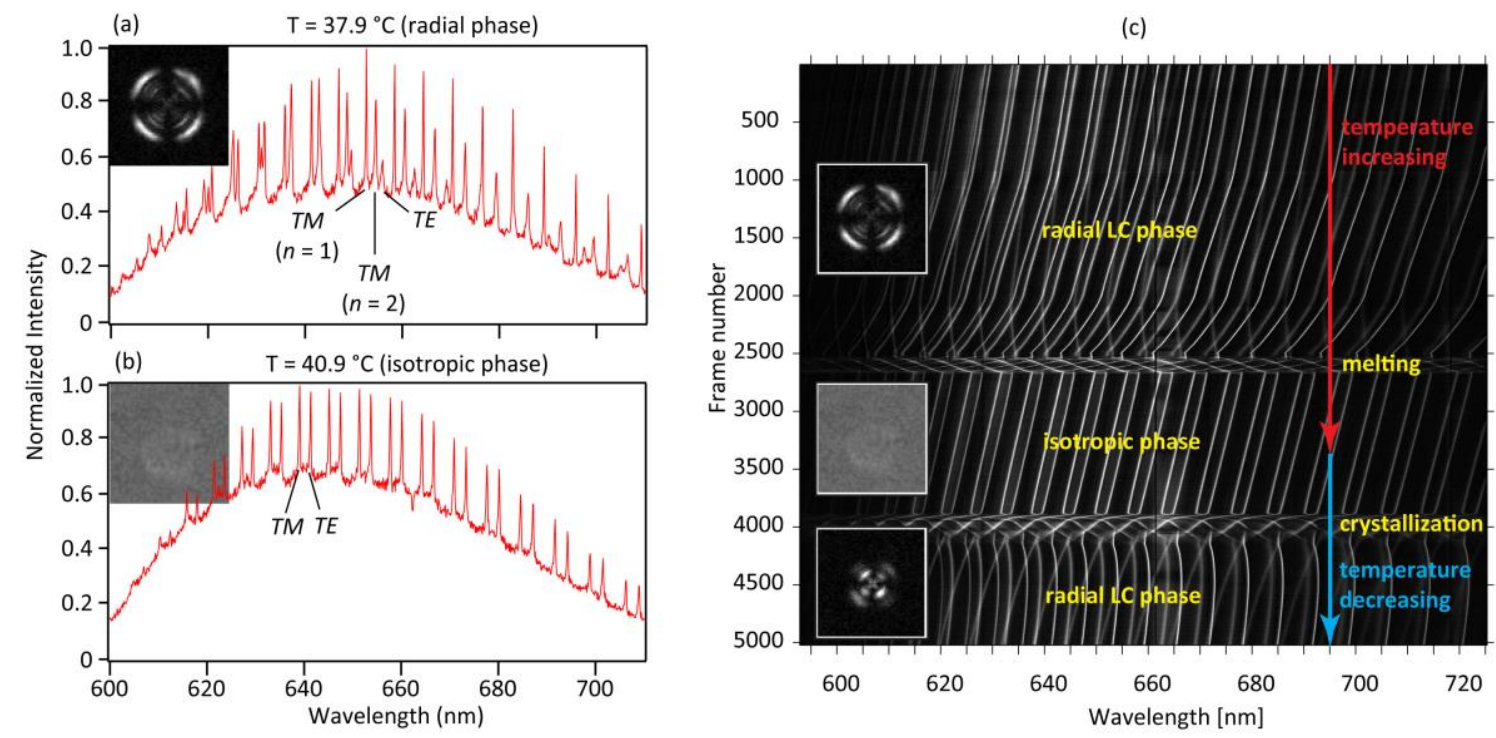

Fig. 1. Tuning of WGM spectra of LC droplets by controlled temperature changes. (a) WGM spectrum of a radial LC droplet. (b) WGM spectrum of an isotropic LC droplet. (c) Complete tuning cycle induced by temperature increase and decrease.

At temperatures below the phase transition point, the droplet is in the birefringent radial LC phase and its emission spectrum features TM-polarized modes of radial orders $n=1,2$ and also TE-polarized modes of the lowest radial order $n=1$ (Fig. 1(a)) [6]. Due to the low contrast between the ordinary refractive index of liquid crystal molecules $n_{o}$ seen by the TE-modes and the refractive index of the surrounding host medium $n_{\text {host }}$, the intensities and Q-factors of the TE-modes are lower than those observed for the TM-modes seeing the extraordinary index $n_{e}$ [7]. Upon melting and transition into the isotropic phase, droplet birefringence resulting from the long-range order of LC molecules vanishes and the droplet observed through crossed polarizer and analyzer becomes practically invisible (Fig. 1(b)). At the same time, TM-polarized modes with $n=2$ disappear and the intensities and Q-factors of TEpolarized modes with radial order $n=1$ become comparable to those of the corresponding TM-modes because both TE- and TM-mode families now see the same average refractive index $n_{\mathrm{LC}}$ of the isotropic phase.

The complete tuning cycle that consists of a temperature increase (frames 1-3400) followed by a temperature decrease (frames 3400-5000) is shown in Fig. 1(c). This figure illustrates good reversibility of temperature-induced spectral tuning; when the temperature is lowered, the droplet transitions back to the radial LC phase and the character of its WGM spectra returns to that shown in Fig. 1(a).

\section{Tuning of emission spectra of LC droplets by external electric field}

Due to the anisotropy of polarizability of liquid crystal molecules, external electric field can be used to change the molecular alignment in the LC phase, thus changing its optical response. In particular, when exposed to an external electric field, LC molecules rotate so that their long axis tends to orient parallel to the applied field. This mechanism can be used for efficient tuning of spectral profile of microscopic cavities based on LC droplets [7].

Fig. 2 summarizes the spectral response of an optically trapped radial LC droplet of diameter approximately 17 $\mu \mathrm{m}$ exposed to external AC electric field of frequency $1 \mathrm{MHz}$ generated by applying AC voltage varying between 0 and $330 \mathrm{Vpp}$ across the sample chamber. Schematics in Fig. 2(a) illustrates the changes in the orientation of LC molecules within the droplet at zero (top) and maximal (bottom) electric field; radial double-headed arrows indicate the direction of the electric field of TM-polarized WGMs. With increasing external electric field, LC molecules gradually turn away from the purely radial orientation and align with the applied external field. Consequently, the extraordinary refractive index $n_{e}$ of LC molecules (given by the polarizability of LC molecules along their long axis) originally seen by the TM-modes starts decreasing towards the ordinary refractive index $n_{o}$ (given by the polarizability of LC molecules along their short axis). This decrease in the effective refractive index of the droplet translates into shortening of the effective optical path length and, thus, shifting of the WGMs to the blue end of the spectrum. The whole process is fully reversible; when the external field drops to zero again, WGMs shift back to their original positions. For the particular droplet shown, spectral tuning range $\Delta \lambda=1.3 \mathrm{~nm}$. 
(a)

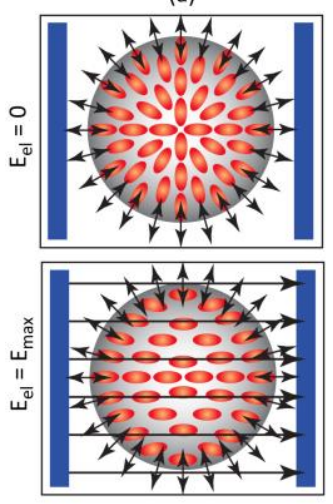

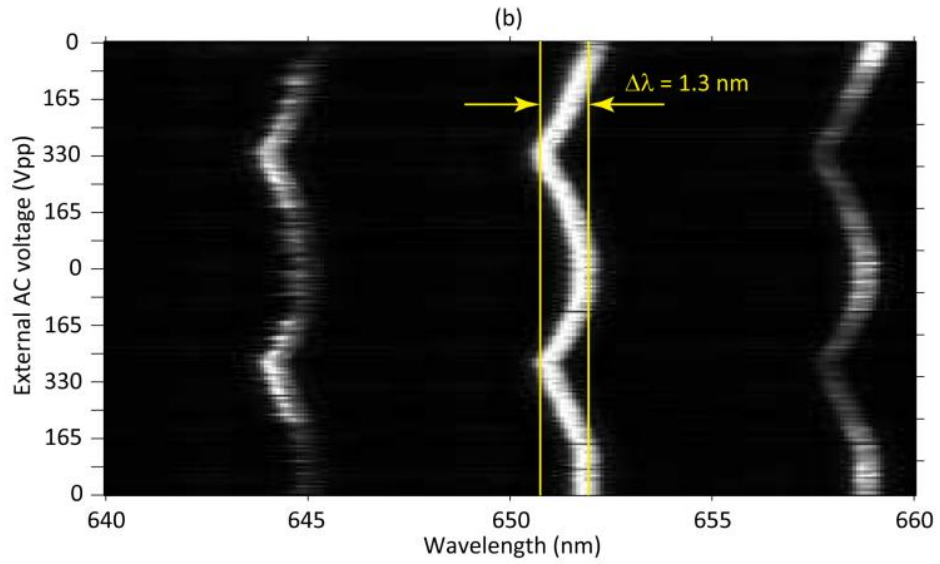

Fig. 2. Tuning of WGM spectra of LC droplets by AC electric field. (a) Schematics of orientation of LC molecules inside a radial LC droplet without (top) and (with) applied external electric field. (b) Complete tuning cycle induced by electric field increase and decrease.

\section{Conclusions}

We have studied tunable optofluidic microcavities formed by dye-doped emulsion droplets of liquid crystals suspended in an aqueous host liquid and confined in optical tweezers. By changing the temperature of the emulsion or applying external electric field to the droplets, we have shown that the WGM spectra of the droplet-based resonators can be largely and almost reversibly tuned in both directions. Residual irreversibility observed in the spectral tuning patterns due to gradual dissolution of the droplet in the host liquid can be potentially reduced or even eliminated by optimizing the composition of the host liquid supporting the droplets (in particular, the type and/or concentration of the used surfactant). The presented results indicate feasibility of this approach for creating miniature largely tunable sources of coherent light for on-chip integration. In addition, spectroscopic measurements with LC droplets subject to external stimuli (e.g. orienting external electric field or controlled changes of the ambient temperature that eventually induce phase transitions in the droplets) can elucidate the physical processes taking place in the droplets, thus contributing to the fundamental research in the field of liquid crystal physics.

\section{Acknowledgement}

We acknowledge funding from TÜBİTAK (114F253), COST Action (MP1205), the Ministry of Education, Youth and Sports of the Czech Republic (LD14069, LO1212), and the European Commission (CZ.1.05/2.1.00/01.0017).

\section{References}

[1] C. Monat, P. Domachuk, and B. J. Eggleton, "Integrated optofluidics: A new river of light," Nat. Photonics 1,106-114 (2007).

[2] X. Fan and I. M. White, “Optofluidic microsystems for chemical and biological analysis,” Nat. Photonics 5, 591-597 (2011).

[3] A. Kiraz, A. Sennaroglu, S. Doganay, M. A. Dundar, A. Kurt, H. Kalaycioglu, and A. L. Demirel, "Lasing from single, stationary, dye-doped glycerol/water microdroplets located on a superhydrophobic surface," Opt. Commun. 276, 145-148 (2007).

[4] S. Nizamoglu, M. C. Gather, and S. H. Yun, “All-biomaterial laser using vitamin and biopolymers,” Adv. Mat. 25, 5943-5947 (2013).

[5] A. Jonas, M. Aas, Y. Karadag, S. Manioglu, S. Anand, D. McGloin, H. Bayraktar, and A. Kiraz, "In vitro and in vivo biolasing of fluorescent proteins suspended in liquid microdroplet cavities," Lab Chip 14, 3093-3100 (2014).

[6] G. C. Righini, Y. Dumeige, P. Feron, M. Ferrari, G. N. Conti, D. Ristic, and S. Soria, "Whispering gallery mode microresonators: fundamentals and applications," Rivista Del Nuovo Cimento 34, 435-488 (2011).

[7] M. Humar, M. Ravnik, S. Pajk, and I. Musevic, "Electrically tunable liquid crystal optical microresonators," Nat. Photonics 3, 595-600 (2009).

[8] M. Humar and I. Musevic, "Surfactant sensing based on whispering-gallery-mode lasing in liquid-crystal microdroplets," Opt. Express 19 , 19836-19844 (2011).

[9] M. Aas, A. Jonas, and A. Kiraz, "Lasing in optically manipulated, dye-doped emulsion microdroplets,” Opt. Commun. 290\}, 183-187 (2013). 